\title{
ARKTOS: A Knowledge Engineering Software Package for Satellite Sea Ice Classification
}

\author{
Leen-Kiat Soh and Costas Tsatsoulis \\ Department of Electrical Engineering and Computer Science \\ University of Kansas, Lawrence, Kansas, USA \\ ITTC, 2291 Irving Hill Road, Lawrence, KS 66044 USA \\ Tel: (785) 864-7764 Fax: (785) 864-0387 E-mail: $\{1 \mathrm{ksoh}$, tsatsoul $\} @$ ittc.ukans.edu
}

\begin{abstract}
In this paper, we describe the knowledge engineering software package of our ARKTOS project. The ARKTOS project involves acquiring knowledge from sea ice experts as visual cues for sea ice features and classification rules and ultimately building an intelligent sea ice classifier. To assist in the knowledge acquisition, evaluation, and refinement phases, we have designed and built three Javabased graphical user interfaces (GUIs): arktosGUI, arktosViewer, and arktosEditor. arktosGUI facilitates feature-based knowledge refinement, focusing the experts' attention on individual features, specific attributes, and rules. It allows inspection of ice features visually, numerically, and symbolically and attribute impact analysis. The objective of arktosViewer is, on the other hand, to enable quick, regionbased evaluation of the classification. It displays annotated, gridded images, maintains a bookkeeping of the evaluation sessions, and allows the experts to record their observation. Finally, the arktosEditor module has a rule indexing and search mechanism to go with its complete rule and threshold editing capabilities. This tool allows the experts to better edit and organize the rule base. The software package helps us design and refine ARKTOS as an intelligent knowledgebased system, and also address research issues in explicit encoding of domain expertise and capture of visual cues and semantics for intelligent image analysis in remote sensing, especially for computer-aided SAR sea ice image analysis.
\end{abstract}

\section{INTRODUCTION}

Our ARKTOS (Advanced Reasoning using Knowledge for Typing Of Sea ice) research performs automated, intelligent SAR sea ice classification. Once it is deployed, it will be incorporated into the flow of the operations to (1) assist geophysicists or photo-interpreters in identifying sea ice classes, and (2) perform pre-processing tasks to help streamline the workflow. In order to emulate human's ability in analyzing image features and combining various source of information, knowledge engineering is an important and evolving stage of the project. The goal of our knowledge engineering process is twofold. First, we aim at acquiring feature-based descriptive knowledge and classification rules from sea ice experts [1]. Second, after extracting and instrumenting such knowledge into a working system, the objective becomes one of refinement and focusing-adjusting the weights of the rules, thresholds of the descriptors, designs of the measurements, and others-to better implement expert knowledge and utilize information from different sources [2] in sea ice classifica- tion. To assist the sea ice experts and developers in knowledge acquisition, evaluation, and refinement phases of the engineering cycle, we have built three graphical user interface (GUI) software modules: arktosGUI, arktosViewer, and arktosEditor. arktosGUI allows users to inspect feature by feature, and attribute by attribute, and how rules are fired and how a classification is derived. arktosViewer allows users to examine regions in an image and record notes on the accuracy and distribution of ARKTOS' sea ice classification. This provides an important feedback tool to developers and researchers in determining quickly what types of images ARKTOS does well or poorly in. arktosEditor allows users to search the rule base, edit rules, and modify attribute thresholds.

These tools provide a visual feedback link to the knowledge engineering effort between the sea ice experts and the developers and a common platform where the two sides can exchange ideas and viewpoints. Therefore, the tools have been useful in designing and refining ARKTOS as an intelligent knowledge-based system, and in the explicit encoding of domain expertise and capture of visual cues and semantics for satellite sea ice classification.

This paper describes the objectives, designs, and implementations of the software modules as part of the knowledge engineering package of ARKTOS.

\section{FEATURE-BASED KNOWLEDGE ENGINEERING}

The objective of this module is to encourage feature-based knowledge refinement, by focusing the user's attention on individual features, specific attributes, and rules. Hence, this software is very useful during the fine-tuning of the ARKTOS rule base. arktosGUI has (1) a feature-based inspection in which users can examine a specific ice feature (e.g., a floe) visually, symbolically, and numerically, and can also analyze the reasoning process that led to the classification of that feature, (2) a compare-and-contrast of different ice features simultaneously, and (3) an attribute impact analysis in which users can view and examine, in color-coded images, the attribute changes resulting from his/her actions.

arktosGUI allows the user (i.e., the sea ice experts) to view the original image, segmented image, classified image, attribute images, and the log of the expert classification of sea ice features. The user may inspect how an image was segmented and determine whether the segmentation was unsatisfactory and affected the subsequent classification, which he or she may also assess by viewing the color-coded classified image. 
Feature-Based Interaction

When the user clicks on a pixel on the original image, arktosGUI outlines visually the ice feature that encompasses the pixel, and simultaneously displays two textual windows. One window contains the raw, numeric measurements associated with the selected ice feature. The other, symbolic, window contains the symbolic facts of the measurements and other sources of information such as SSM/I maps, landmasks, and ancillary data. In addition, the symbolic window lists the rules that have fired leading to the classification of the particular ice feature and the log of the combined mass of evidence and the belief and plausibility measures of the classification. This facility allows the user to view visually what a particular ice feature looks like and how ARKTOS numerically and symbolically views that feature. Thus, the user can compare ARKTOS' views to his or hers. For example, if the user thinks that the feature is not an elongated region but ARKTOS thinks so, then the user might be motivated to change the design of the "elongatedness" measurement and its thresholds. In addition, the symbolic window allows the user to see how the brain of ARKTOS works by following its trail of fired rules. The user can thus identify problematic rules for changes.

Moreover, the user is able to compare and contrast different features by clicking on different pixels on the original image. Each outlined ice feature is accompanied by a pair of textual windows. Thus, the user can check to see whether two visually similar features are also symbolically similar in ARKTOS' interpretation, or whether two visually different features result in two distinctive descriptions.

Indeed, this feature-based interaction has been utilized intensively to refine the threshold values used to convert numeric measurements into symbolic facts and the creation and modification of our classification rules. Fig. 1 shows an example of arktosGUI.

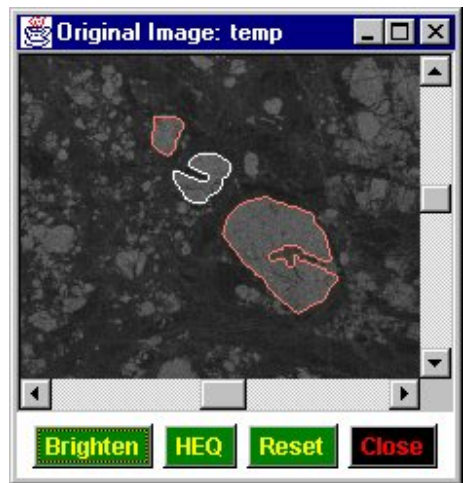

Fig. 1 An example of arktosGUI. Feature-based interaction allows the user to select and view ice features individually.

\section{Attribute Impact Analysis}

This software feature allows the user to select a particular attribute and views the distribution of its attribute values of all features in the image. For example, suppose the user selects "return", the average intensity of an ice feature, which takes four values: black, dark, gray, and bright. arktosGUI subsequently displays a feature-based image, with each feature colored accordingly, all dark features are colored blue, dark features are red, gray features are green, bright features are white, and features with unknown returns are gray. This enables the user to perform an effective review of the attribute's correctness. The user can change the thresholds of an attribute, re-run the ARKTOS program, and view the impact of that change on the screen. Fig. 2 shows an example of the attribute impact analysis feature.

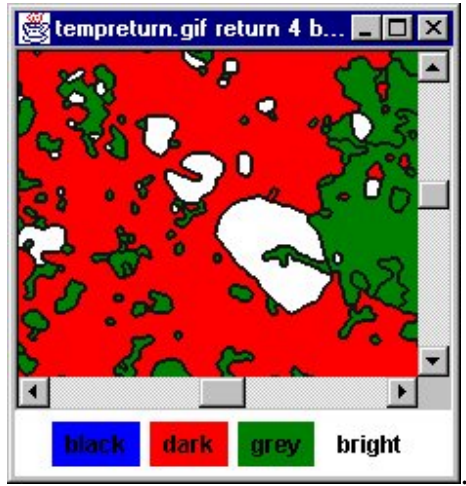

Fig. 2 An example of arktosGUI. An attribute image (attribute is "return") that shows how each ice feature fares. For example, the selected features (in Fig. 1) are all bright features, surrounded by dark and gray water and ice mixtures.

\section{REGION-BASED KNOWLEDGE ENGINEERING}

The objective of this module is to facilitate region-based evaluation, helping the user concentrate on regions instead of individual ice features and on ice type distributions instead of the classification of a particular feature. Therefore, this module is important in broadbase evaluation of the ARKTOS system and in determining which images ARKTOS classifies well or poorly, in a large database of images. arktosViewer has (1) a region-based inspection in which users can examine annotated, gridded area to assess whether the classification results are acceptable, (2) a bookkeeping of the user's evaluation sessions, and (3) a text-based recording of the user's feedback and reviews.

arktosViewer divides an image into $4 \times 4$ regions and computes for each region its ice type distributions. It then imposes a grid onto the image and displays the distributions to the user.

Region-Based Interaction

The user may survey the gridded and annotated image and, if satisfactory, simply click the "acceptable" button to score the image. Otherwise, the user may click on regions that he or she thinks are incorrectly classified. The regions are highlighted as a result, and the user is able to toggle off the selection anytime they want. In addition, the actions are automatically time-stamped and written to a text file for bookkeeping tractability. Fig. 3 shows an example of arktosViewer. 


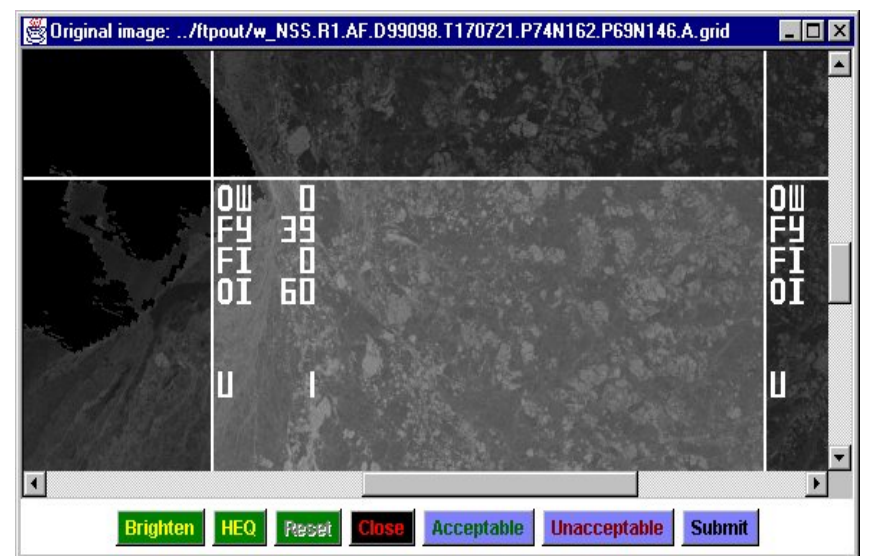

Fig. 3 An example of arktosViewer. ARKTOS classifies the highlighted region to $0 \%$ open water, $39 \%$ first year ice, $0 \%$ fast ice, $60 \%$ old ice, and $1 \%$ unknown.

\section{Annotator}

Together with the display module, arktosViewer also features a free-text window for the user. The user can type in notes and observations, and the text is recorded and tagged with the image in question. This allows additional information to be transferred from the user (the sea ice experts) to the developers.

\section{KNOWLEDGE BASE EDITING}

The main goal of arktosEditor is to allow better editing of the knowledge base. This module has (1) full object-based rule editing and threshold editing capabilities, and (2) a rule indexing and search mechanism. It features indexed, attribute-based, and conditioned search and filtering, editing of all aspects (ID, conditions, weights, classifications, and text description) of a rule, and editing of the thresholds used to convert numeric measurements to symbolic facts. Hence, this module is important in organizing and modifying our knowledge bases.

\section{Rule Editor}

The rule editor allows the user to delete an old rule, modify an existing rule, and create a new rule. Each rule can have a combination of up to ten different attributes. On the screen, the possible values of each attribute are on display when it is selected. For example, suppose the user is creating a new rule and he or she wants to include the attribute "size". Immediately after the selection, the user is able to click on the trailing menu to select one of the choices: small, average, and large. This design thus relieves the user from having to memorize or look up some manuals to obtain the set of possible values for each attribute. Fig. 4 shows an example of arktosEditor.

\section{Threshold Editor}

The threshold editor allows the user to modify the threshold values of the attributes.

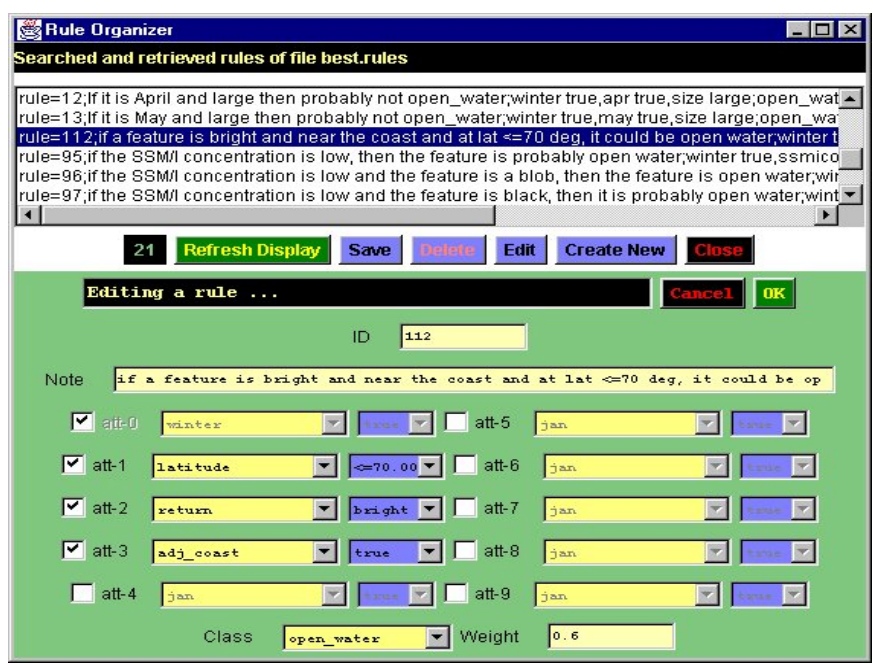

Fig. 4 An example of arktosEditor. An existing rule is selected and its attribute-value pairs are automatically displayed and the user can change its ID, text description, attributevalue pairs, classification, and weight.

\section{CONCLUSIONS}

The knowledge engineering software package of ARKTOS is implemented in Java and C. It runs on both the Windows NT and the UNIX operating systems. The package has helped us in acquiring, refining, and evaluating our existing knowledge base, from the design and implementation of attributes to the writing of a classification rule, and has been successfully incorporated into our ARKTOS sea ice classification system. We also see the package as a useful tool in capturing visual cues and semantics for intelligent image analysis in remote sensing. Current work includes improving the computational ties and data and file management among the three software modules.

\section{ACKNOWLEDGMENTS}

We would like to thank Denise Generis of NRL at Stennis Space Center, Mary Ruth Keller and Cheryl Bertoia of the National Ice Center (NIC) for the continuous evaluation of ARKTOS and its knowledge engineering software package. This research work is supported in part by a Naval Research Laboratory research grant, contract number N00014-95-C6038.

\section{REFERENCES}

[1] L.-K. Soh, C. Tsatsoulis, T. Bowers, and A. Williams, "Representing Sea Ice Knowledge in Dempster-Shafer Belief System," IGARSS '98, Seattle, WA, pp. 22342236, July 1998.

[2] L.-K. Soh and C. Tsatsoulis. "Multisource Data and Knowledge Fusion for Intelligent SAR Sea Ice Classification," IGARSS'99, Hamburg, Germany, pp. 68-70, July 1999. 